\title{
CHALLENGES IN SIMULATING BEAM DYNAMICS OF DIELECTRIC LASER ACCELERATION
}

\author{
U. Niedermayer ${ }^{1,2 *}$,
}

A. Adelmann ${ }^{7}$, R. Aßmann ${ }^{4}$, S. Bettoni ${ }^{7}$, D. S. Black ${ }^{2}$, O. Boine-Frankenheim ${ }^{1}$, P. N. Broaddus ${ }^{2}$, R. L. Byer ${ }^{2}$, M. Calvi ${ }^{7}$, H. Cankaya ${ }^{4,11,15}$, A. Ceballos ${ }^{2}$, D. Cesar ${ }^{10}$, B. Cowan ${ }^{9}$, M. Dehler ${ }^{7}$, H. Deng ${ }^{2}$, U. Dorda ${ }^{4}$, T. Egenolf ${ }^{1}$, R. J. England ${ }^{3}$, M. Fakhari ${ }^{4}$, A. Fallahi ${ }^{11}$, S. Fan ${ }^{2}$, E. Ferrari ${ }^{5,7}$, F. Frei ${ }^{7}$, T. Feurer ${ }^{12}$, J. Harris ${ }^{2}$, I. Hartl ${ }^{4}$, D. Hauenstein ${ }^{7}$, B. Hermann ${ }^{7,12}$, N. Hiller ${ }^{7}$, T. Hirano ${ }^{2}$, P. Hommelhoff ${ }^{6}$, Y.-C.Huang ${ }^{13}$, Z. Huang ${ }^{2}$, T. W. Hughes ${ }^{2}$, J. Illmer 6 , R. Ischebeck ${ }^{7}$, Y. Jiang ${ }^{2}$, F. Kärtner ${ }^{4}, 11,15$, W. Kuropka ${ }^{4,15}$, T. Langenstein ${ }^{2}$, Y. J. Lee ${ }^{8}$, K. Leedle ${ }^{2}$, F. Lemery ${ }^{4}$, A. Li ${ }^{6}$, C. Lombosi ${ }^{7}$, B. Marchetti ${ }^{4}$, F. Mayet ${ }^{4,15}$, Y. Miao ${ }^{2}$, A. Mittelbach ${ }^{6}$, P. Musumeci $^{10}$, B. Naranjo ${ }^{10}$, A. Pigott ${ }^{2}$, E. Prat ${ }^{7}$, M. Qi ${ }^{8}$, S.Reiche $^{7}$, L. Rivkin ${ }^{5,7}$, J. Rosenzweig ${ }^{10}$, N. Sapra ${ }^{2}$, N. Schönenberger ${ }^{6}$, X. Shen ${ }^{10}$, R. Shiloh ${ }^{6}$, E.Skär ${ }^{1}$, E. Simakov ${ }^{14}$, O. Solgaard ${ }^{2}$,

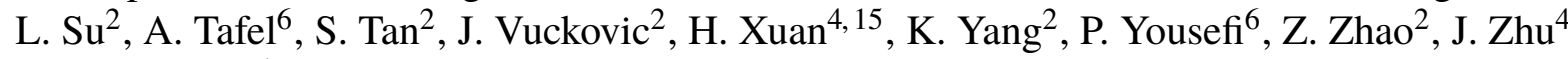

${ }^{1}$ Technische Universität Darmstadt, 64289 Darmstadt, Germany

2 Stanford University, Stanford, CA 94305, USA

${ }^{3}$ SLAC National Accelerator Laboratory, Menlo Park, CA 94025

${ }^{4}$ Deutsches Elektronen-Synchrotron, D-22607 Hamburg, Germany

5 École Polytechnique Fédérale de Lausanne, CH-1015 Lausanne, Switzerland

${ }^{6}$ Friedrich-Alexander Universität Erlangen-Nürnberg, 91058 Erlangen, Germany

${ }^{7}$ Paul Scherrer Institut CH-5232 Villigen, Switzerland

${ }^{8}$ Purdue University, West Lafayette, IN 47907, USA

9 Tech-X Corporation, Boulder, CO 80303, USA

${ }^{10}$ University of California, Los Angeles, CA 90095, USA

${ }^{11}$ Center for Free-Electron Laser Science, 22607 Hamburg, Germany

${ }^{12}$ Universität Bern, Switzerland

13 Nat. Tsing Hua University, Taiwan

${ }^{14}$ Los Alamos National Laboratory, USA

15 Universität Hamburg, 22761 Hamburg, Germany

\section{Abstract}

Dielectric Laser Acceleration (DLA) achieves the high$\tau_{\text {est }}$ gradients among structure-based electron accelerators.

The use of dielectrics increases the breakdown field limit,

and thus the achievable gradient, by a factor of at least 10

in comparison to metals. Experimental demonstrations of DLA in 2013 led to the Accelerator on a Chip International Program (ACHIP), funded by the Gordon and Betty Moore o Foundation. In ACHIP, our main goal is to build an accelerator on a silicon chip, which can accelerate electrons from below $100 \mathrm{keV}$ to above $1 \mathrm{MeV}$ with a gradient of at least $100 \mathrm{MeV} / \mathrm{m}$. For stable acceleration on the chip, magnet$\Rightarrow$ only focusing techniques are insufficient to compensate the strong acceleration defocusing. Thus spatial harmonic and Alternating Phase Focusing (APF) laser-based focusing techniques have been developed. We have also developed the $\$$ simplified symplectic tracking code DLAtrack6D, which makes use of the periodicity and applies only one kick per DLA cell, which is calculated by the Fourier coefficient of the synchronous spatial harmonic. Due to coupling, the *niedermayer@temf.tu-darmstadt.de

Fourier coefficients of neighboring cells are not entirely independent and a field flatness optimization (similarly as in multi-cell cavities) needs to be performed. The simulation of the entire accelerator on a chip by a Particle In Cell (PIC) code is possible, but impractical for optimization purposes. Finally, we have also outlined the treatment of wake field effects in attosecond bunches in the grating within DLAtrack6D, where the wake function is computed by an external solver.

\section{INTRODUCTION}

The Accelerator on a Chip International Program (ACHIP) [1], funded by the Gordon and Betty Moore Foundation in the period between 2015 and 2020, aims to explore Dielectric Laser Acceleration (DLA). This nascent acceleration scheme provides the highest gradients among structurebased (non-plasma, non-vacuum, etc.) electron accelerators and thus allows reduction of the size of high energy electron accelerators significantly. The principle of DLA relies on the inverse Smith-Purcell (or the inverse Cerenkov effect) and was first proposed in 1962 [2,3]. In 2013, the accel- 
eration of relativistic electrons was first demonstrated at SLAC with a gradient of more than $250 \mathrm{MeV} / \mathrm{m}$ in a $\mathrm{SiO}_{2}$ double grating structure driven by a $800 \mathrm{~nm}$ Ti:Sapphire laser [4]. In the same setup, the gradient was later increased to $690 \mathrm{MeV} / \mathrm{m}$ [5]. Also in 2013, strongly sub-relativistic electrons $(27.7 \mathrm{keV})$ were accelerated by the group at FAU Erlangen with a gradient of $25 \mathrm{MeV} / \mathrm{m}$ using a single grating structure at the third spatial harmonic [6]. The group at Stanford University used a silicon dual pillar structure to accelerate $96 \mathrm{keV}$ electrons with a gradient of more than $200 \mathrm{MeV} / \mathrm{m}$ [7] and a similar experiment at $30 \mathrm{keV}$ with few-cycle laser pulses was done at FAU Erlangen [8]. An example of such a dual pillar structure is shown in Fig. 1. These schemes all utilize laterally incident lasers with polar-
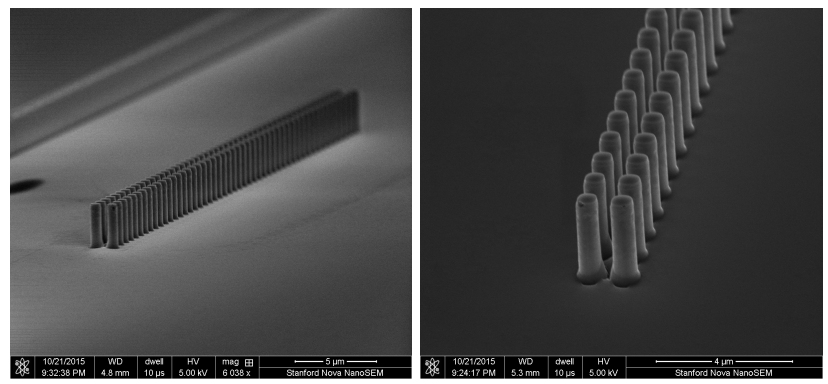

Figure 1: Scanning Electron Microscope (SEM) pictures of a dual pillar acceleration structure.

ization in the electron beam direction, thus the accelerating near field is a standing wave. It is also possible to use longitudinally coupled (traveling wave) structures, see [9] for details and a general overview.

The goal of ACHIP is to build an accelerator as sketched in Fig. 2, which can accelerate electrons from electrostatic sources $(<100 \mathrm{keV})$ to above $1 \mathrm{MeV}$. A second goal is to make use of the accelerator by exploring the options of DLA based deflection (see e.g. [10]), which can potentially lead to laser driven undulators [11-13].

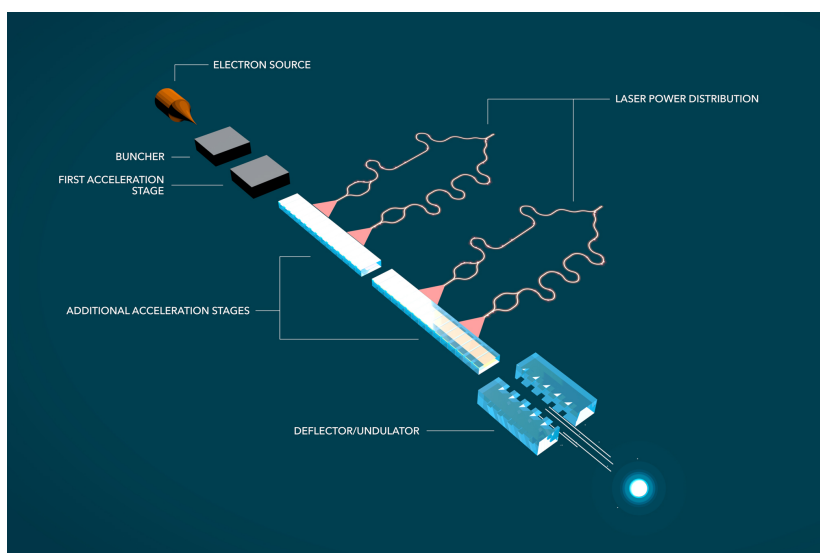

Figure 2: Sketch of the goals of the ACHIP collaboration.

Different materials have been investigated for DLA [14]. In order to achieve the highest gradient, the material-specific damage threshold fluence

$$
F_{\text {dam }}>F=\frac{P \Delta t}{A},
$$

where $P / A$ is the laser intensity and $\Delta t$ is the pulse length, is approached, but must not be exceeded. Note that the dependence on the laser wavelength and pulse length can be strongly nonlinear, see e.g. [15] for an in-depth discussion and more empirical data. Moreover, if the laser travels a longer distance through the material, the nonlinear phase shift also needs to be considered [16]. In general, a shorter pulse allows for a higher gradient at the same fluence. Moreover, a high band gap material as e.g $\mathrm{SiO}_{2}$ will have a higher damage threshold and a low band gap material such as Silicon has a lower damage threshold but a higher refractive index.

For the sub-relativistic experiments in the ACHIP collaboration we mostly use $\lambda_{0}=2 \mu \mathrm{m}$ femtosecond laser pulses, generated by Optical Parametric Amplifiers (OPA) or by novel Tm or Ho-Tm fiber laser amplifiers currently under development. The electron source needs to provide ultra-low emittance, particularly at low energy. For a lossless sub$100-\mathrm{keV}$ injection into a DLA operating at $2 \mu \mathrm{m}$, geometric emittances smaller than $0.1 \mathrm{~nm}$ are required [17]. Different emitters are available to produce these low emittances, e.g. [18], or see [19] for an overview. At these emittances, the achievable charge is quite small at the moment. We hope to achieve higher average charge in the future by increasing both the repetition rate and the single microbunch charge.

\section{DIFFERENT MEANS OF LASER COUPLING}

All the DLA experiments performed so far have used free space laser coupling. At longer interaction length, it is necessary to provide symmetric fields, such that there is no coherent deflection force. There are different means to obtain symmetric fields in the acceleration channel, the simplest is to illuminate the structure symmetrically from both sides with equal phase and polarization. If this is impractical from the optics point of view, the fields of a single side drive laser can also be symmetrized by using a Bragg mirror on the chip [20,21].

The laser fluence on each DLA cell can be reduced by shortening the pulse and tilting the pulse front [22,23], such that it remains synchronous with a few electron bunches over a distance (or duration) much longer than a single DLA cell is illuminated, see Fig. 3. Practically, the pulse front tilt

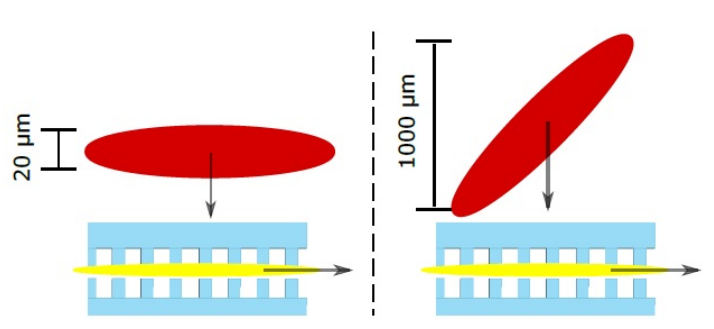

Figure 3: Flat vs. tilted laser pulse. At roughly the same pulse length for each grating cell, the interaction length is significantly increased. Picture adapted from [22]. 
E can be achieved, for example, by a prism or by a reflection

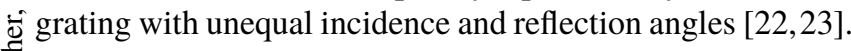

Another option to increase the interaction length in the acZ celerator structure is an on-chip waveguide system [24], see Fig. 4. This supplies different parts of the accelerator struc-

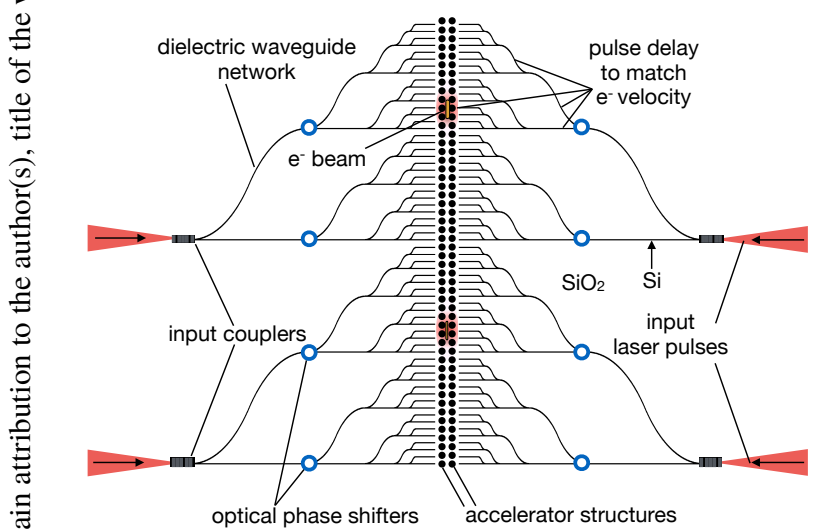

Figure 4: On-chip waveguide laser power delivery system. Picture adapted from [24].

ture with the appropriate phase and group delay. Moreover, a different, higher damage threshold and lower refractive index material can be used to convey higher laser fluence in the waveguides, which can be split in to many waveguides before coupling to the high refractive index accelerator structure. Using this technique, it is possible to produce a similar illumination pattern as would be obtained by pulse front tilt in free space.

\section{FIELD COMPUTATION FOR A SINGLE DLA CELL}

The laser field computation of a single DLA cell using periodic boundary conditions is not a challenge, since its electrical length $L / \lambda$ is on the order of one. It can be simu-

$\dot{m}$ lated by various techniques such as Finite Difference / Finite

Integration Time Domain (FD/FI TD) codes [25, 26], Finite

U Difference Frequency Domain (FDFD) codes [27], or Finite

Element Frequency Domain (FEFD) codes [26,28]. These $\checkmark$ can be combined with various optimization techniques, in gै order to find structures with highest gradient, lowest field inside the material, or highest bandwidth. Of course, these optimization goals compete, such that an optimum can only be found in the sense of a Pareto-front. Simple DLA structures can also be designed from a physical point of view, such o that maximum electric field modulation at the synchronous o harmonic is achieved, which results in Bragg cavity structures $[28,29]$. A more mathematical approach is rather to use adjoint methods to perform large-scale, gradient-based optimization of the full permittivity distribution [30]. However, these methods tend to generate non-inutitive device geometries and sometimes require additional constraints to create fabricable structures. Moreover, adjoint methods have also been used for other parts of the integrated DLA, such as grating couplers [31].

\section{BEAM DYNAMICS SIMULATIONS IN DLATRACK6D}

We will summarize and slightly add to DLAtrack6D, the one kick per cell tracking approach originally conceived in [29]. The kicks are sufficiently described by one complex coefficient per DLA cell, where the longitudinal and transverse dependencies are derived analytically. Although the derivation holds true only for strict periodicity, small deviations can be accepted within reasonable error. Also fringe fields are not included, even in practice they should be reduced as much as possible. The effect of fringe fields is however strongly dependent on the quality factor of the structure, usually determined by the available bandwidth.

Starting from the longitudinal energy gain, the kicks in all directions are computed and then used for symplectic tracking. The energy gain of an electron with charge $q=-e$ can be written as function of the time domain electric field $E_{z}$, its Frequency Domain (FD) phasor $\underline{E}_{z}$, or by means of spatial Fourier series in periodic DLA structures

$$
\begin{aligned}
\Delta W(x, y, s) & =q \int_{-\lambda_{g z} / 2}^{\lambda_{g z} / 2} E_{z}(x, y, z ; t=(z+s) / v) \mathrm{d} z \\
& =q \lambda_{g z} \operatorname{Re}\left\{e^{2 \pi i \frac{s}{\beta \lambda_{0}}} \underline{e}_{m}(x, y)\right\} .
\end{aligned}
$$

Here the spatial Fourier coefficient is computed as

$$
\underline{e}_{m}(x, y)=\frac{1}{\lambda_{g z}} \int_{-\lambda_{g z} / 2}^{\lambda_{g z} / 2} \underline{E}_{z}(x, y, z) e^{i m \frac{2 \pi}{\lambda g z} z} \mathrm{~d} z .
$$

The above relation holds only if the Wideroe condition $\lambda_{g z}=$ $m \beta \lambda_{0}$ is fulfilled, where $\lambda_{g z}$ is the grating period and $\beta$ is the velocity in units of $c$. In the following we will restrict the arbitrary integer spatial harmonic $m$ to $m=1$, which usually has the strongest amplitude $\underline{e}_{1}$.

The transverse kicks can be obtained by exploiting the known transverse dependency of $e_{1}(x, y)$ on the transverse coordinates. From the Panofsky-Wenzel theorem [32], conveniently written as

$$
\nabla^{\prime} \times \Delta \vec{p}(x, y, s)=0,
$$

where the relative gradient is $\nabla^{\prime}=\left(\partial_{x}, \partial_{y},-\partial_{s}\right)$, we obtain under the synchronicity condition

$$
\Delta \vec{p}_{\perp}(x, y, s)=-\frac{\lambda_{g z}^{2}}{2 \pi} q \frac{1}{\beta c} \operatorname{Im}\left\{e^{2 \pi i \frac{s}{\beta \lambda_{0}}} \nabla_{\perp} \underline{e}_{1}(x, y)\right\} .
$$

For a symmetric laser illumination the transverse dependencies can be written as

$$
\underline{e}_{1}(x, y)=\underline{e}_{1}(0,0) \cosh \left(i k_{y} y\right) e^{i k_{x} x},
$$

where $k_{x}$ includes the option of a tilt of the grating or the laser incidence, $k_{z}=2 \pi /\left(\beta \lambda_{0}\right)$, and $k_{y}=$ $\pm \sqrt{\left(2 \pi / \lambda_{0}\right)^{2}-k_{z}^{2}-k_{x}^{2}}$. For a grating tilt angle $\alpha$ (see Fig. 5) we obtain [29] $k_{x}=k_{z} \tan (\alpha)$ and for a laser tilt angle $\vartheta$ (keeping the polarization parallel to the electron beam) we obtain $k_{x}=k_{z} \sin (\vartheta)$. After some manipulations, we finally 


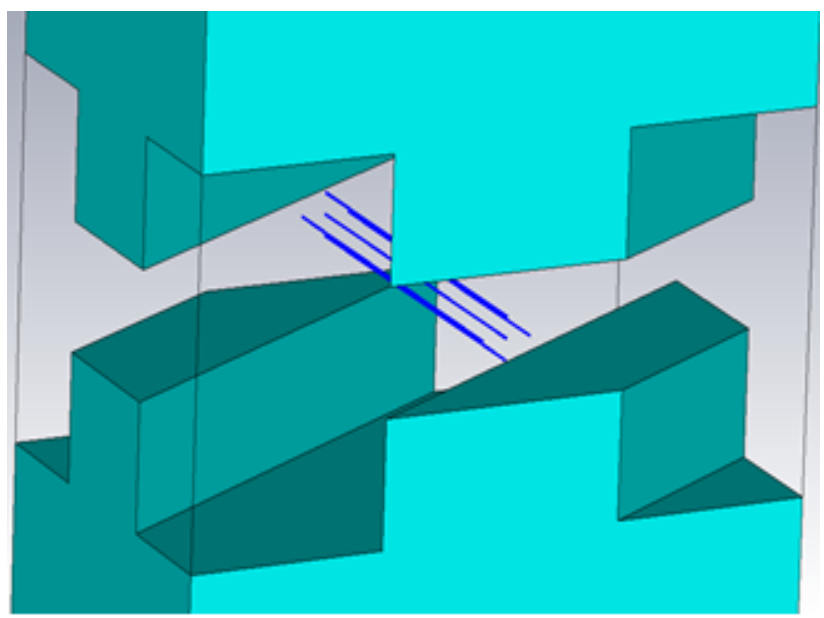

Figure 5: Unit cell of a tilted grating (tilt angle $\alpha$ ). The blue lines indicate possible electron trajectories, the laser is incident from top and bottom, with polarization in electron beam direction and possible tilt angle $\vartheta$.

obtain the kicks [29]

$$
\begin{aligned}
& \Delta x^{\prime}=-\frac{q \lambda_{0}}{p_{z 0} c} \tan (\alpha) \cosh \left(i k_{y} y\right) \operatorname{Re}\left\{\underline{e}_{1} e^{i \varphi+i k_{x} x}\right\} \\
& \Delta y^{\prime}=\frac{-i k_{y} \lambda_{0}^{2} q \beta}{2 \pi p_{z 0} c} \sinh \left(i k_{y} y\right) \operatorname{Im}\left\{\underline{e}_{1} e^{i \varphi+i k_{x} x}\right\} \\
& \Delta \delta=\frac{q \lambda_{g z}}{\gamma m_{e} c^{2}} \operatorname{Re}\left\{\underline{e}_{1}\left(\cosh \left(i k_{y} y\right) e^{i \varphi+i k_{x} x}-e^{i \varphi_{s}}\right)\right\} .
\end{aligned}
$$

In case of an anti-symmetric illumination, i.e. a $\pi$ phase shift between the two laser beams, the transverse dependence is

$$
\underline{e}_{1}(x, y)=\underline{e}_{1}(0,0) \sinh \left(i k_{y} y\right) e^{i k_{x} x}
$$

and the kicks are accordingly

$$
\begin{aligned}
& \Delta x^{\prime}=-\frac{q \lambda_{0}}{p_{z 0} c} \tan (\alpha) \sinh \left(i k_{y} y\right) \operatorname{Re}\left\{\underline{e}_{1} e^{i \varphi+i k_{x} x}\right\} \\
& \Delta y^{\prime}=\frac{-i k_{y} \lambda_{0}^{2} q \beta}{2 \pi p_{z 0} c} \cosh \left(i k_{y} y\right) \operatorname{Im}\left\{\underline{e}_{1} e^{i \varphi+i k_{x} x}\right\} \\
& \Delta \delta=\frac{q \lambda_{g z}}{\gamma m_{e} c^{2}} \operatorname{Re}\left\{\underline{e}_{1} \sinh \left(i k_{y} y\right) e^{i \varphi+i k_{x} x}\right\} .
\end{aligned}
$$

Note that in the sinh-mode, the energy gain of the synchronous particle is always zero, since the longitudinal electric field vanishes in center of the channel. The sinh-mode can be used as a diagnostic, in order to convert a temporal profile into an angle distribution profile [33], which is usually referred to as beam streaking.

The symplectic one-kick-per-cell tracking is independent of the realization of the kick functions and reads

$$
\left(\begin{array}{c}
x \\
x^{\prime} \\
y \\
y^{\prime} \\
\varphi \\
\delta
\end{array}\right)^{(n+1)}=\left(\begin{array}{c}
x \\
A x^{\prime}+\Delta x^{\prime} \\
y \\
A y^{\prime}+\Delta y^{\prime} \\
\varphi \\
\delta+\Delta \delta\left(\varphi_{\mathrm{s}}\right)
\end{array}\right)^{(n)}+\left(\begin{array}{c}
\lambda_{g z} x^{\prime} \\
0 \\
\lambda_{g z} y^{\prime} \\
0 \\
-\frac{2 \pi}{\beta^{2} \gamma^{2}} \delta \\
0
\end{array}\right)^{(n+1)}
$$

with the tracking variables in paraxial approximation

$$
\begin{aligned}
x^{\prime} & =\frac{p_{x}}{p_{z 0}}, \quad \Delta x^{\prime}=\frac{\Delta p_{x}(x, y, \varphi)}{p_{z 0}}, \\
y^{\prime} & =\frac{p_{y}}{p_{z 0}}, \quad \Delta y^{\prime}=\frac{\Delta p_{y}(x, y, \varphi)}{p_{z 0}}, \\
\varphi & =2 \pi \frac{s}{\lambda_{g z}}, \quad \delta=\frac{W-W_{0}}{W_{0}}, \\
\Delta \delta & =\frac{\Delta W(x, y, \varphi)-\Delta W\left(0,0, \varphi_{\mathrm{s}}\right)}{W_{0}},
\end{aligned}
$$

where $W_{0}=\gamma m_{e} c^{2}$ and $p_{z 0}=\beta \gamma m_{e} c$. The adiabatic damping of the transverse emittance is described by

$$
A^{(n)}=\frac{(\beta \gamma)^{(n+1)}}{(\beta \gamma)^{(n)}}=1+\left[\frac{\lambda_{0} q \operatorname{Re}\left\{e^{i \varphi_{s}} \underline{e}_{1}\right\}}{\beta \gamma m_{e} c^{2}}\right]^{(n)} .
$$

While keeping the synchronicity condition by appropriately chirping the structure, the acceleration ramp can be written as

$$
W(N)=W(0)+q \sum_{n=1}^{N} \lambda_{g}^{(n)} \operatorname{Re}\left\{e_{1}^{(n)} e^{i \varphi_{s}^{(n)}}\right\} .
$$

We note that chirping the structure length while maintaining constant phase $\arg \left(\underline{e}_{1}\right)$ is possible by correcting the phase drift with another parameter in the structure design [20].

DLAtrack6D is written in Matlab [34], it is based on a phase space structure that allows for vectorized updates in each DLA cell. The code, together with a brief manual, will be made available to the community soon.

\section{ELECTRON BEAM FOCUSING}

Optical near field accelerators cannot rely on magnetic focusing only, since the small scale of the near field requires sub-micron beam sizes which in turn would require magnetic field strengths unachievable by conventional quadrupole magnets [35]. Thus a laser-based focusing scheme is required to make DLA scalable.

Two different options have been proposed for focusing with the phase dependent transverse laser fields. In 2012 the group at UCLA has proposed spatial harmonic focusing [36]. Stability of the electron beam could be predicted by means of retracting ponderomotive forces due to non-synchronous harmonics, while the synchronous harmonic serves for acceleration. However, the beam envelope at given emittance could not be determined more accurately than in the smooth approximation. Moreover, the focusing harmonic needs to be quite strong (much stronger than the accelerating harmonic), which puts a constraint on the choice of materials and pulse length due to the damage threshold fluence. In particular, this scheme has been implemented with $\mathrm{SiO}_{2}$ structures at relativistic energies [37].

Recently, Niedermayer et al. have proposed Alternating Phase Focusing for DLA [17]. Here, we work only with a single spatial harmonic, i.e. $\underline{e}_{1}$, but its phase can be changed by means of fractional period drift elements. The hereby generated longitudinal/transversal alternating focusing gradients 
can be integrated in the Courant-Snyder sense. Thus a scal$\approx$ able scheme is obtained, where about half (dependent on the synchronous phase) of the synchronous harmonic is translated to acceleration gradient. The required pre-bunching on the optical scale can be obtained with the same scheme, see [17]. Due to the exact integration of the lattice (and of thus a precise determination of the beam envelope) and the $t \leftarrow$ efficient translation of incident field to acceleration gradient, this scheme is particularly suited for sub-relativistic DLAs -working with high refractive index Silicon structures that have a rather low damage threshold. An outline of such a structure is depicted in Fig. 6.

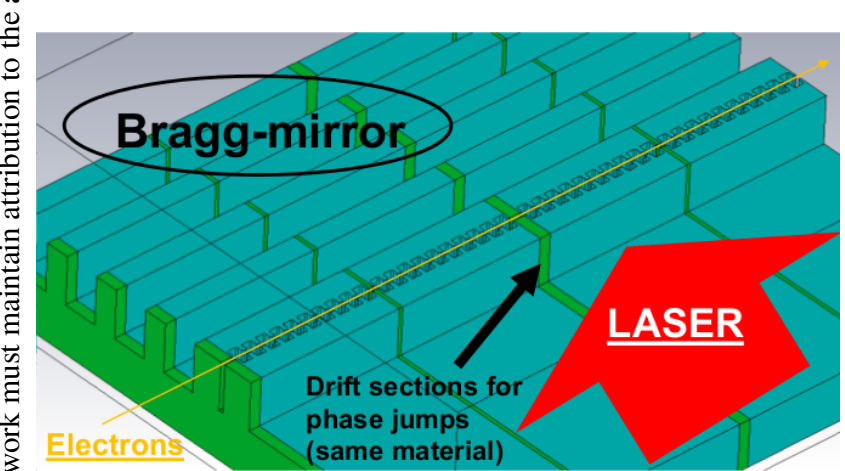

Figure 6: Outline of an APF DLA structure, picture adapted from [29].

\section{FULL SCALE SIMULATION TECHNIQUES}

For full scale tracking and PIC simulations we mostly use $\dot{\infty}$ the codes CST Studio Suite [26] and VSim [25]. A full scale

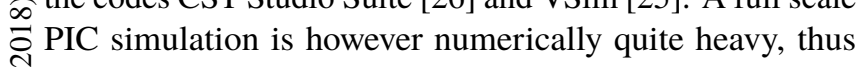
(9) we prefer to do this only for finished designs and rely on DLAtrack6D for design studies. In CST, we have the conve. nient option to calculate the fields in either in TD or FD and store them as frequency domain phasors, i.e. one complex $\dot{r}$ number per mesh edge. Note that storing in FD does not necessary mean computation in FD; in fact, FD simulations become intractable as the simulation size becomes large, so

\section{.}
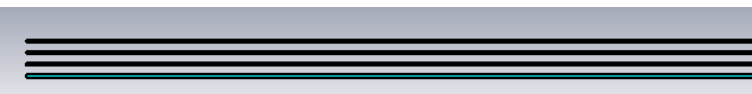

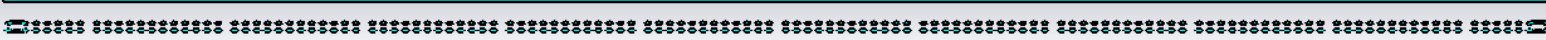

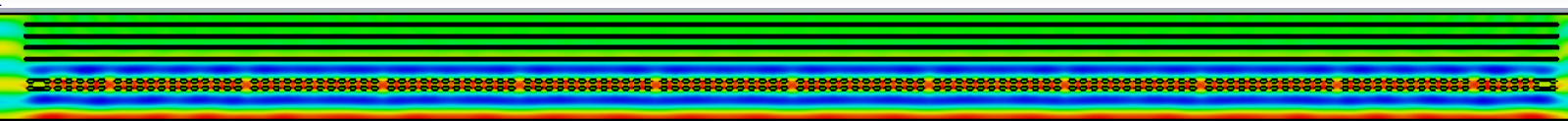

2.2

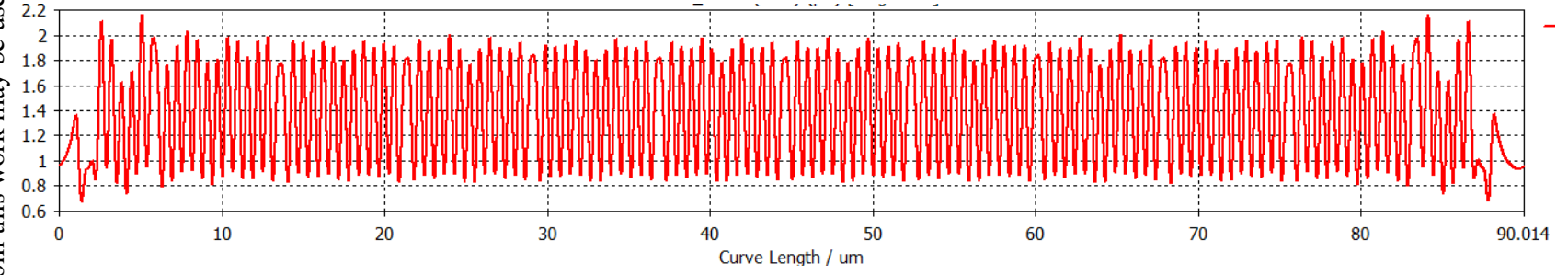

Figure 7: Alternating Phase Focusing single laser beam transport structure (top), normalized longitudinal electric field phasor magnitude with laser from bottom (center) and on axis field flatness plot (bottom), obtained by CST MWS in TD. 
Moreover, another crucial optimization is to tune the Bragg mirror distance such that the fields in the channel become symmetric. After the optimized fields have been determined, electron tracking can be done both in DLAtrack6D and full PIC codes. Figure 8 shows how the fraction of electrons transported through the structure is dependent on the laser field strength (DLAtrack6D).
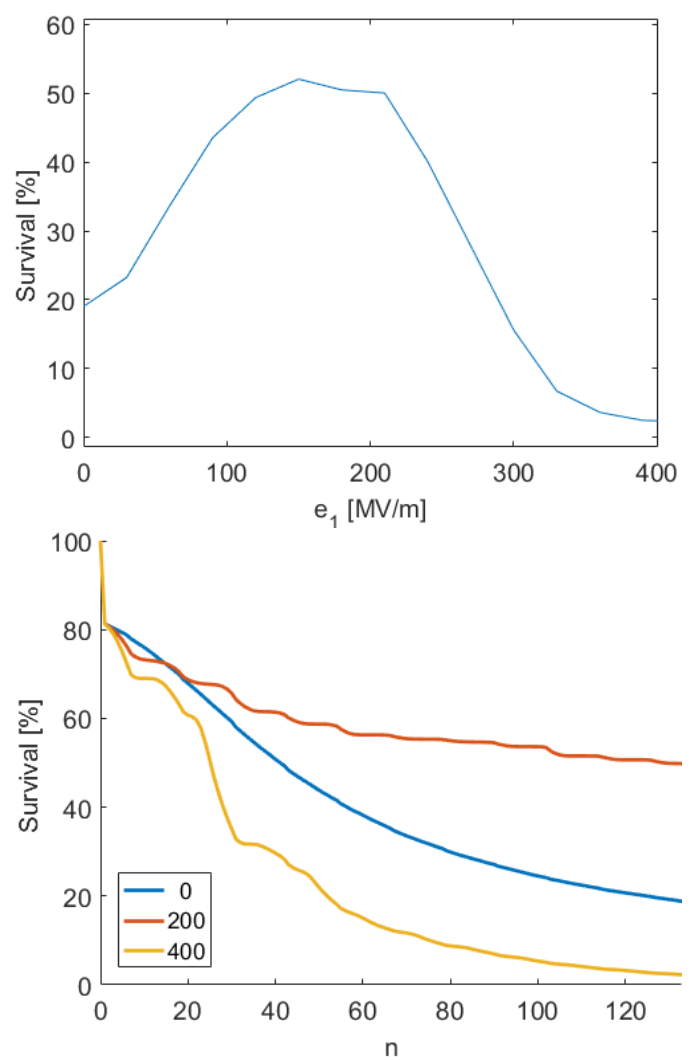

Figure 8: Transmission rate as function of laser field strength (top) and electron loss as function of DLA cell index for $e_{1}=(0,200,400) \mathrm{MV} / \mathrm{m}$ laser field strength (bottom).

The minimal beta-function is obtained at roughly $200 \mathrm{MV} / \mathrm{m}$, where maximum transmission occurs. Beyond that point, the beam is first over-focused, and eventually leaves the area of stability. The reason for the losses at the matched $e_{1}$ is the geometric emittance of $0.3 \mathrm{~nm}$ (Gaussian distribution initially), which is larger than the acceptance of the structure. Once saturated at about 50\%, the electrons can be transported over an arbitrary distance, which is limited only by the defocusing in the direction of the pillar height. Roughly the same results are obtained by tracking in the CST PIC solver.

In succession to $[33,39]$, we plan a two stage buncheraccelerator or buncher-streaker experiment, see Fig. 9. Here we use dual drive from two stages, with independent phase control and independent amplitude control of lasers 1 and 2. The first DLA stage is normally run in cosh-mode and serves as a buncher. The second stage can be either run in cosh-mode for acceleration, or in sinh-mode for streaking. Replacing the first (buncher) structure by an APF-type buncher, see [17], will allow us to obtain short bunches with

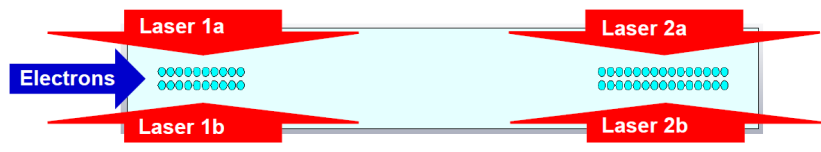

Figure 9: Combined buncher and accelerator/streaker DLA.

low energy spread. This gives the opportunity to observe both coherent streaking and coherent acceleration at the same setup on a spectrometer screen.

Additionally to the low energy experiment, there are also high energy experiments planned. Most prominently, we will use the $3.2 \mathrm{GeV}$ beam at SwissFEL at PSI to inject into a DLA. Due to the extremely small geometric emittance at such high energy, this injection will be almost lossless. Additionally, at such energy the deflection and acceleration defocusing is rather small. Therefore, we are restricted only by the conventional electron optics (Rayleigh length) and the available laser pulse energy, which can be cast in a tilted pulse. Details of the outlined experiments can be found in $[40,41]$.

Moreover, relativistic energy experiments are also outlined at the SINBAD facility at DESY, where an inverse FEL undulator together with a chicane will be employed for optical-scale bunching of the beam before it is injected into the DLA. Driving the DLA with the same laser as is used for seeding the inverse FEL allows for the precise phase control required for coherent acceleration [42].

The group at UCLA aims for a 2-cm long DLA experiment at about $5 \mathrm{MeV}$ injection energy at the Pegasus facility [43] Challenges are that focusing of the beam is still required and also a slight chirp needs to be imprinted to account for the slightly sub-relativistic velocity. Creating both the focusing harmonics and the chrip is planned to be achieved by a strictly periodic $\mathrm{SiO}_{2}$ grating fed by a tilted laser pulse that is modulated by a Spatial Light Modulator, see e.g. [44].

\section{CURRENT STATUS AND OUTLOOK}

We are now able to perform start-to-end simplified simulations of larger DLA chips with DLAtrack6D. For full scale 3D PIC or tracking simulations a cluster computer is required. The experiments performed at the moment can still be simulated well in 2D by available PIC codes. However, it is expected that the structure lengths will soon significantly increase.

One option for efficient large scale PIC for DLA would be a moving window code, which discretizes only the comoving environment of a few electron micro-bunches. The rest of the structure contains neither electrons nor laser energy, since we strongly restrict the interaction region by applying the pulse front tilt or other means of selective synchronized illumination.

The charge we accelerate in current DLA experiments is mostly rather low. However, at particular high energy experiments as e.g. at PSI, the entire beam is put through the small aperture of the DLA. We expect to see wake field effects here for the first time. Simulations of wake field effects are already in place [45]. We outline to integrate (a) . 政 을

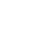
裀 然 .

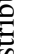

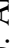
क्ष i (2)

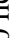

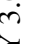
. 
13th Int. Computational Accelerator Physics Conf.

оं ISBN: $978-3-95450-200-4$

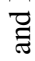

linear and non-linear wake kicks from precomputed wake

$\ddot{\otimes}$ functions into DLAtrack6D as well. With this we will be

able to properly predict the strength of beam loading effects

and longitudinal and transverse beam instabilities in longer

DLA structures. dation (Grant No. GBMF4744 to Stanford). U.N. acknowlक्ष edges also the funding by the German Federal Ministry of Education and Research (Grant No. FKZ:05K16RDB). B.C. 壱 (TechX) acknowledges usage of NERSC, a U.S. Department $\approx$ of Energy Office of Science User Facility operated under

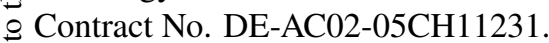

\section{REFERENCES}

[1] ACHIP website: https://achip.stanford.edu

[2] K. Shimoda, Applied Optics, vol. 1, pp. 33, 1962.

[3] A. Lohmann, IBM Technical Note, 169, 1962.

[4] E. A. Peralta et al., "Demonstration of electron acceleration in a laser-driven dielectric microstructure", Nature, vol. 503, pp. 91, 2013. doi : 10.1038/nature12664

[5] K. P. Wootton et al., Opt. Lett., vol. 41, pp. 2696, 2016.

[6] J. Breuer and P. Hommelhoff, Physical Review Letters, vol. 111, pp. 134803, 2013.

[7] K. J. Leedle, A. Ceballos, H. Deng, O. Solgaard, R. Fabian Pease, R. L. Byer, and J. S. Harris, Optics Letters, vol. 40, pp. 4344, 2015.

[8] M. Kozak et al., NIM A, vol. 865, pp. 84-86, 2016.

[9] R. J. England et al., Reviews of Modern Physics, vol. 86, pp. 34, 2014.

[10] J. McNeur et al., Optica, vol. 5, issue 6, pp. 687-690, 2018. doi:10.1364/OPTICA.5.000687

[11] T. Plettner and R. L. Byer, Phys. Rev. STAccel. Beam, vol. 11, pp. 030704, 2008.

[12] T. Plettner and R. L. Byer, Nucl. Instrum. Methods Phys. Res. A, vol. 593, pp. 63, 2008.

[13] T. Plettner, R. Byer, C. McGuinness, and P. Hommelhoff, Phys. Rev. ST Accel. Beams, vol. 12, pp. 101302, 2009.

[14] K. Soong, R. L. Byer, E. R. Colby, R. J. England, and E. A. Peralta, AIP Conference Proceedings, vol. 1507, pp. 511,2012

[15] P. Pronko et al., Physical Review B, vol. 58, pp. 2387, 1998. [16] D. Cesar et al., Nat. Comm. Phys., vol. 1, pp. 46, 2018.

[17] U. Niedermayer, T. Egenolf, O. Boine-Frankenheim, and P. Hommelhoff, accepted for publication in Phys. Rev. Lett., 2018. arXiv: 1806.07287

[18] D. Ehberger, J. Hammer, M. Eisele, M. Krueger, J. Noe, A. Hogele, and P. Hommelhoff, Physical Review Letters, vol. 114, pp. 1, 2015.
ICAP2018, Key West, FL, USA JACoW Publishing doi:10 . 18429/JACoW - ICAP2018-MOPLGO1

[19] A. Feist et al., Ultramicroscopy, vol. 176, pp. 63, 2017.

[20] U. Niedermayer, O. Boine-Frankenheim, and T. Egenolf, Journal of Physics, Conference Series, vol. 874, 2017.

[21] P. Yousefi et al. https://arxiv.org/abs/1801.05234

[22] D. Cesar, J. Maxson, P. Musumeci, X. Shen, R. J. England, and K. P. Wootton, SLAC-PUB-17180, 2018.

[23] M. Kozak et al., Journal of Applied Physics, vol. 124, 2018. doi:10.1063/1.5032093

[24] T. W. Hughes et al., Physical Review Applied, vol. 9, pp. 54017, 2018

[25] VSim, www.txcorp.com

[26] CST Studio Suite 2018, www.cst. com

[27] W. Shin and S. Fan, Optics Express, vol. 21, no. 19, pp. 2257822595,2013

[28] T. Egenolf, O. Boine-Frankenheim, and U. Niedermayer, Journal of Physics: Conference Series, vol. 874, 2017. doi : $10.1088 / 1742-6596 / 874 / 1 / 012040$

[29] U. Niedermayer, T. Egenolf, and O. Boine-Frankenheim, Phys. Rev. AB, vol. 20, pp. 111302, 2017.

[30] T. Hughes et al., Optics Express, vol. 25, Issue 13, 2017. doi:10.1364/OE.25.015414

[31] N. Sapra et al., https://arxiv.org/abs/1808.07630

[32] W. K. H. Panofsky and W. A. Wenzel, Review of Scientifc Instruments, vol. 27, pp. 31, 1956.

[33] K. J. Leedle, D. S. Black, Y. Miao, K. E. Urbanek, A. Ceballos, H. Deng, J. S. Harris, O. Solgaard, and R. L. Byer, Optics Letters, vol. 43, pp. 2181, 2018.

[34] MathWorks, Matlab, 2016.

[35] A. Ody, P. Musumeci, J. Maxson, D. Cesar, R. J. England, and K. P. Wootton, NIM A, vol. 865, pp. 75, 2017.

[36] B. Naranjo, A. Valloni, S. Putterman, and J. B. Rosenzweig, Physical Review Letters, vol. 109, pp. 1, 2012.

[37] D. Cesar, J. Maxson, P. Musumeci, X. Shen, R. J. England, K. P. Wootton, and S. Tan, arXiv preprint accepted for publication in Optics Express, 1804.00634, 2018.

[38] D. Black, K. Leedle, Y. Miao, U. Niedermayer, R.L.Byer, and O. Solgaard, unpublished.

[39] M. Kozak et al., Nat. Comm., vol. 8, pp. 14342, 2017.

[40] E. Prat et al., NIM A, vol. 865, pp. 87-90, 2017.

[41] E. Ferrari et al., NIM A, vol. 907, pp. 244-247, 2018.

[42] F. Mayet et al., accepted for publication in NIM-A, 2018. doi:10.1016/j.nima.2018.01.088

[43] D. Cesar, P. Musumeci and R. J. England, in Proc. of $A A C 2018$, IEEE Conf Series, to appear.

[44] ThorLabs EXULUS-HD1 Spatial Light Modulator www. thorlabs.com

[45] T. Egenolf, U. Niedermayer, and O. Boine-Frankenheim in Proc. of AAC2018, IEEE Conf Series, to appear. 